Research Article

\title{
Lie Symmetry Analysis for the General Classes of Generalized Modified Kuramoto-Sivashinsky Equation
}

\author{
Rong Qi, ${ }^{1}$ Muhammad Mobeen Munir ${ }^{D},{ }^{2}$ Nazish Younas, ${ }^{3}$ Muhammad Idrees, ${ }^{3}$ \\ and Jia-Bao Liu $\mathbb{D}^{4}$ \\ ${ }^{1}$ School of Computer and Data Engineering, Bengbu College of Technology and Business, Bengbu 233000, China \\ ${ }^{2}$ Department of Mathematics, University of the Punjab, Quaid-e-Azam Campus, Lahore, Pakistan \\ ${ }^{3}$ Department of Mathematics, University of Education, Lahore, Pakistan \\ ${ }^{4}$ School of Mathematics and Physics, Anhui Jianzhu University, Hefei 230601, China
}

Correspondence should be addressed to Muhammad Mobeen Munir; mmunir@ue.edu.pk

Received 19 August 2021; Accepted 30 September 2021; Published 25 October 2021

Academic Editor: Fanglei Wang

Copyright (c) 2021 Rong Qi et al. This is an open access article distributed under the Creative Commons Attribution License, which permits unrestricted use, distribution, and reproduction in any medium, provided the original work is properly cited.

Lie symmetry analysis of differential equations proves to be a powerful tool to solve or at least reduce the order and nonlinearity of the equation. Symmetries of differential equations is the most significant concept in the study of DE's and other branches of science like physics and chemistry. In this present work, we focus on Lie symmetry analysis to find symmetries of some general classes of KS-type equation. We also compute transformed equivalent equations and some invariant solutions of this equation.

\section{Introduction}

Symmetry has been a source of inspiration as a powerful tool in the formulation of the laws of the universe. A great number of physical phenomena is transformed into differential equations. Lie symmetry analysis can change the given differential equation into an equivalent form which is easier to solve. In the analysis of differential equations, the symmetry group approach is quite useful. Galois's use of finite groups to solve algebraic equations of degrees two, three, and four, as well as to prove that the general polynomial equation of degrees larger than four could not be solved by radicals, served as the paradigm for this application [1-7]. The symmetry group approach is well-known for its importance in the field of differential equations analysis. Sophus Lie is credited with the invention of group categorization methods and the theoretical basis for the Lie groups.

There are many different methods for computing the symmetries of differential equations. But Lie symmetry analysis is the best because it is a systematic and algorithmic procedure that does not take into account any guesses or approximations. The principal paper on Lie symmetry is
[1], in which Lie demonstrated that a linear 2D, 2nd-order PDE admits at most three boundary invariance group. $\mathrm{He}$ processed the maximal invariance group of the onedimensional heat conductivity and used this analysis to compute its explicit solutions. Symmetry reduction is a leading strategy for resolving nonlinear PDEs. Ovisiannikov made a substantial contribution in persisting with these techniques. He presented the strategy of partially invariant solutions [2, 3]. In this work, he gave a methodology that is based on the idea of group called the equivalence group. Gazizov and Ibragimov [8] tracked down the total symmetry analysis of the onedimensional Black-Scholes model. Shu-Yong and Feng-Xiang, [9] discussed about the connection between the form invariance and Lie symmetry of nonholonomic framework. Buckwar and Luchko [4] initiated the study of symmetry group of scaling transformation for PDEs of fractional order. Yan et al. [6] performed Lie symmetry analysis and fundamental similarity reductions for the coupled Kuramoto-Sivashinsky(KS) equations. Bozhkov and Dimas [10] computed the conversation laws and group classification for generalized 2D KS equation. Nadjafikhah and Ahangari [7] determined the Lie symmetries and reduction for the two-dimensional damped 


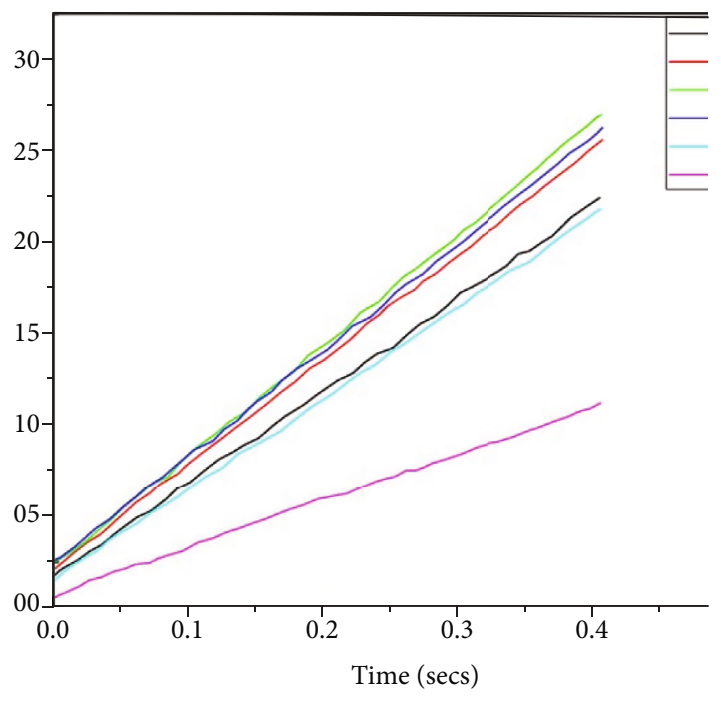

FIGURE 1: Flame front position.

Kuramoto-Sivashinsky ((2D) DKS) equation. Najafikhah and Ahangari also computed Lie symmetry of 2D generalized Kuramoto-Sivashinsky (KS) equation in [11]. The onedimensional modified KS-type equation is

$$
u_{t}+u_{x x}+u_{x x x x}+(\lambda-1) u_{x}^{2}-\sigma u_{x x}^{2}=0,
$$

Chou [12] determined the solution of the cauchy problem for the MKS equation and also computed the solvability with the help of the blow up theorem.

In the present paper, we deal with the generalized modified one-dimensional Kuramoto-Sivashinsky (GMKS) type equation and determine the symmetry algebra by using Lie symmetry analysis. In particular, we want to find the optimal system and similarity solutions corresponding to some special cases of GMKS equation. The GMKS type equation is given as

$$
f(u) u_{t}+u_{x x}+u_{x x x x}+(\lambda-1) u_{x}^{2}-\sigma u_{x x}^{2}=0 .
$$

We seek the Lie symmetry algebras for this GMKS equations for $f(u)=u^{n}, f(u)=e^{n u}$, and $f(u)=e^{u^{n}}$ where $\lambda$ and $\sigma$ are arbitrary constant and $\lambda \neq 1$. For $\lambda=1$, equation (2) is proposed in [13]. Its second derivative satisfies an equation of Cahn-Hilliard type in [14]. This equation has various applications as physical models in biofluids, mechanics, and liquids. In equation (2), $u$ is the velocity function, $x$ is space parameter, and $t$ is time variable. This equation can also be derived from a model in the continuity equation by fitting a suitable function [15]. Actually, the Kuramoto-Sivashinsky equation gives the change of the position of a flame front (Figure 1). It shows the flame front position against time for horizontally propagating methane flame, the movement of a fluid going down a vertical wall, or a spatially uniform oscillating chemical reaction in a homogeneous medium [16]. This equation is also helpful to display solitary pulses in a falling slender film [17]. Figure 2 shows the schematic representation of the flow showing a film flowing vertically down, subjected to an electric field imposed across electrodes separated by a distance $d$.

\section{Lie Symmetry of Generalized Modified Kuramoto-Sivashinsky Equation}

In this part, we compute our main results.

Consider one parameter local Lie group of transformation for the independent factors $x, t$ and dependent factor $u$ as follows:

$$
\begin{aligned}
& x^{*}=x+\delta \alpha(x, t, u)+O\left(\delta^{2}\right), \\
& t^{*}=t+\delta \beta(x, t, u)+O\left(\delta^{2}\right), \\
& u^{*}=u+\delta \gamma(x, t, u)+O\left(\delta^{2}\right),
\end{aligned}
$$

in which $\delta \in \mathbb{R}$ is the parameter.

Proposition 1. For all $n \geq 1, n \in N$, the algebra of symmetries of

$$
u^{n} u_{t}+u_{x x}+u_{x x x x}+(\lambda-1) u_{x}^{2}-\sigma u_{x x}^{2}=0,
$$

is 2-dimensional Abelian Lie algebra.

Proof. The general infinitesimal generator (symmetries) is

$$
H=\alpha(x, t, u) \partial_{x}+\beta(x, t, u) \partial_{t}+\gamma(x, t, u) \partial_{u} .
$$

The derivation of $n$th prolongation of $H$

$$
\operatorname{pr}^{n} H=H+\sum_{i=1}^{q} \sum_{P} \gamma_{i}^{P}\left(x, u^{n}\right)\left(\frac{\partial}{\partial u_{P}^{i}}\right)
$$

interprets the relating jet space $Q^{n} \subset X \times U^{n}$, where $q$ is a dependent variables, and $P=\left(P_{1}, P_{2}, \cdots P_{k}\right)$ with $1 \leq P_{k} \leq p$, $1 \leq k \leq n$ and

$$
\gamma_{i}^{P}\left(x, u^{n}\right)=D_{P}\left(\gamma_{i}-\sum_{l=1}^{p} \alpha^{l} u_{l}^{i}\right)+\sum_{l=1}^{p} \alpha^{l} u_{P, l}^{i},
$$

where $p$ is an independent variable and $u_{l}^{i}=\partial u^{i} / \partial u^{l}$ and $u_{P, l}^{i}=\partial u_{P}^{i} / \partial x^{l}$.

The fourth-order prolongation of $H$ is

$$
\begin{aligned}
& \operatorname{Pr}^{(4)} H= H+\gamma^{x} \frac{\partial}{\partial u_{x}}+\gamma^{t} \frac{\partial}{\partial u_{t}}+\gamma^{x x} \frac{\partial}{\partial u_{x x}}+\gamma^{x t} \frac{\partial}{\partial u_{x t}} \\
&+\gamma^{t t} \frac{\partial}{\partial u_{t t}}+\gamma^{x x x} \frac{\partial}{\partial u_{x x x}}+\gamma^{x x t} \frac{\partial}{\partial u_{x x t}}+\gamma^{x t t} \frac{\partial}{\partial u_{x t t}} \\
&+\gamma^{t t t} \frac{\partial}{\partial u_{t t t}}+\gamma^{x x x x} \frac{\partial}{\partial u_{x x x x}}+\gamma^{x x x t} \frac{\partial}{\partial u_{x x x t}} \\
&+\gamma^{x x t t} \frac{\partial}{\partial u_{x x t t}}+\gamma^{x t t t} \frac{\partial}{\partial u_{x t t t}}+\gamma^{t t t t} \frac{\partial}{\partial u_{t t t t}}, \\
& \operatorname{pr}^{4}\left[u^{n} u_{t}+u_{x x}+u_{x x x x}+(\lambda-1) u_{x}^{2}-\sigma u_{x x}^{2}\right] \equiv 0 \bmod (2), \\
& n u^{n-1} \gamma^{t}+\gamma^{x x}+\gamma^{x x x x}+2(\lambda-1) u_{x} \gamma^{x}-2 \sigma u_{x x} \gamma^{x x} \equiv 0 \bmod (2) .
\end{aligned}
$$




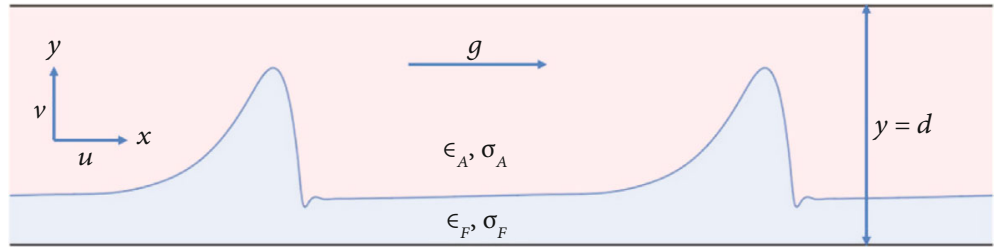

FIGURE 2: Schematic representation of the flow showing a film flowing vertically down.

TABle 1: Commutator table.

\begin{tabular}{lcc}
\hline$[. ., .]$. & $H_{1}$ & $H_{2}$ \\
\hline$H_{1}$ & 0 & 0 \\
$H_{2}$ & 0 & 0 \\
\hline
\end{tabular}

TABLE 2: Adjoint table.

\begin{tabular}{lll}
\hline ad & $H_{1}$ & $H_{2}$ \\
\hline$H_{1}$ & $H_{1}$ & $H_{2}$ \\
$H_{2}$ & $H_{1}$ & $H_{2}$ \\
\hline
\end{tabular}

We can calculate $\gamma^{t}, \gamma^{x}, \gamma^{x x}$, and $\gamma^{x x x x}$ from equation (7) such that

$$
\begin{gathered}
\gamma^{t}=D_{t}\left(\gamma-\alpha u_{x}-\beta u_{t}\right)+\alpha u_{x t}+\beta u_{t t}, \\
\gamma^{x}=D_{x}\left(\gamma-\alpha u_{x}-\beta u_{t}\right)+\alpha u_{x x}+\beta u_{x t}, \\
\gamma^{x x}=D_{x}^{2}\left(\gamma-\alpha u_{x}-\beta u_{t}\right)+\alpha u_{x x x}+\beta u_{x x t}, \\
\gamma^{x x x x}=D_{x}^{4}\left(\gamma-\alpha u_{x}-\beta u_{t}\right)+\alpha u_{x x x x x}+\beta u_{x x x x t},
\end{gathered}
$$

where $D_{x}$ and $D_{t}$ are total derivatives. $\square$

Putting all the above in equation (9) and eliminating $u_{t}$ by using the relation $u_{t}=1 / u^{n}\left(\sigma u_{x x}^{2}-(\lambda-1) u_{x}^{2}-u_{x x}-\right.$ $\left.u_{x x x x}\right)$, we get a polynomial equation containing the different differentials of $u$. Equating the coefficient of $u$ to zero, which are some derivatives of $\alpha, \beta$, and $\gamma$, it gives the total set of determining equations.

$$
\alpha_{x}=\alpha_{t}=\alpha_{u}=0, \beta_{x}=\beta_{t}=\beta_{u}=0, \gamma=0 .
$$

This gives

$$
\alpha(x, t, u)=0, \beta(x, t, u)=0, \gamma(x, t, u)=0 .
$$

This implies that the Lie group (algebra) of infinitesimal generators of equaution (2) is comprised of two vector fields:

$$
\begin{aligned}
& H_{1}=\partial_{x}, \\
& H_{2}=\partial_{t} .
\end{aligned}
$$

The commutator table of the Lie group for equation (2) is given as in Table 1 ,

The adjoint table of infinitesimal symmetries for equation (2) is given as in Table 2,
In this case, we have only two different basis for a Lie algebra of symmetries.

Hence, this shows that the group of symmetries of equation (2) is two dimensional and tables ensure that it is abelian.

Proposition 2. For all $n>1$ and $n \in N$, the group of symmetries of

$$
e^{u^{n}} u_{t}+u_{x x}+u_{x x x x}+(\lambda-1) u_{x}^{2}-\sigma u_{x x}^{2}=0
$$

is two-dimensional abelian.

Proof. The infinitesimal generator is

$$
X=\alpha(x, t, u) \partial x+\beta(x, t, u) \partial t+\gamma(x, t, u) \partial u .
$$

In order to find the symmetry group of equation (14), we have to apply invariance condition that is $X^{(4)}(5) \equiv 0$ mod (6) on equation .(14) where $X^{(4)}$ is the fourth-order prolongation of $X$ given as

$$
\begin{aligned}
\operatorname{Pr}^{(4)} X= & X+\gamma^{x} \frac{\partial}{\partial u_{x}}+\gamma^{t} \frac{\partial}{\partial u_{t}}+\gamma^{x x} \frac{\partial}{\partial u_{x x}}+\gamma^{x t} \frac{\partial}{\partial u_{x t}} \\
& +\gamma^{t t} \frac{\partial}{\partial u_{t t}}+\gamma^{x x x} \frac{\partial}{\partial u_{x x x}}+\gamma^{x x t} \frac{\partial}{\partial u_{x x t}}+\gamma^{x t t} \frac{\partial}{\partial u_{x t t}} \\
& +\gamma^{t t t} \frac{\partial}{\partial u_{t t t}}+\gamma^{x x x x} \frac{\partial}{\partial u_{x x x x}}+\gamma^{x x x t} \frac{\partial}{\partial u_{x x x t}} \\
& +\gamma^{x x t t} \frac{\partial}{\partial u_{x x t t}}+\gamma^{x t t t} \frac{\partial}{\partial u_{x t t t}}+\gamma^{t t t t} \frac{\partial}{\partial u_{t t t t}} .
\end{aligned}
$$

After applying an invariance condition on equation (14), we get

$$
\begin{aligned}
& n u^{n-1} e^{u^{n}} \gamma^{t}+\gamma^{x x}+\gamma^{x x x x}+2(\lambda-1) u_{x} \gamma^{x} \\
& -2 \sigma u_{x x} \gamma^{x x} \equiv 0 . \bmod (2) .
\end{aligned}
$$

We can calculate $\gamma^{t}, \gamma^{x}, \gamma^{x x}$, and $\gamma^{x x x x}$ from equation (7) such that

$$
\begin{gathered}
\gamma^{t}=D_{t}\left(\gamma-\alpha u_{x}-\beta u_{t}\right)+\alpha u_{x t}+\beta u_{t t}, \\
\gamma^{x}=D_{x}\left(\gamma-\alpha u_{x}-\beta u_{t}\right)+\alpha u_{x x}+\beta u_{x t}, \\
\gamma^{x x}=D_{x}^{2}\left(\gamma-\alpha u_{x}-\beta u_{t}\right)+\alpha u_{x x x}+\beta u_{x x t}, \\
\gamma^{x x x x}=D_{x}^{4}\left(\gamma-\alpha u_{x}-\beta u_{t}\right)+\alpha u_{x x x x x}+\beta u_{x x x x t},
\end{gathered}
$$

where $D_{x}$ and $D_{t}$ are total derivatives. 
TABLe 3: Commutator table.

\begin{tabular}{lcc}
\hline$[. ., .]$. & $X_{1}$ & $X_{2}$ \\
\hline$X_{1}$ & 0 & 0 \\
$X_{2}$ & 0 & 0 \\
\hline
\end{tabular}

TABLe 4: Adjoint table.

\begin{tabular}{lll}
\hline$a d$ & $X_{1}$ & $X_{2}$ \\
\hline$X_{1}$ & $X_{1}$ & $X_{2}$ \\
$X_{2}$ & $X_{1}$ & $X_{2}$ \\
\hline
\end{tabular}

After putting all the above in equation (17), and eliminating $u_{t}$ by using the relation $u_{t}=1 / e^{u^{n}}\left(\sigma u_{x x}^{2}-(\lambda-1) u_{x}^{2}\right.$ $\left.-u_{x x}-u_{x x x x}\right)$, we get a polynomial equation containing the different differentials of $u$. Equating the coefficient of $u$ to zero, which are some derivatives of $\alpha, \beta$, and $\gamma$, it gives the total set of determining equations, as given by

$\alpha_{x}=\alpha_{t}=\alpha_{u}=0, \beta_{x}=\beta_{t}=\beta_{u}=0, \gamma=0$,

that gives

$$
\alpha(x, t, u)=0, \beta(x, t, u)=0, \gamma(x, t, u)=0 .
$$

This implies that the Lie group (algebra) of infinitesimal generators of equation (9) comprises two vector fields. Following, Table 3 gives the commutator table as

$$
\begin{aligned}
& X_{1}=\partial_{x}, \\
& X_{2}=\partial_{t} .
\end{aligned}
$$

The commutator table of the Lie group for equation (14) is given in Table 3,

The adjoint table of infinitesimal symmetries for equation (14) is given in Table 4,

In this case, we have only two different basis for Lie algebra.

Hence, this shows that the group of symmetries of equation (14) is two-dimensional abelian.

2.1. Symmetry Algebra for $e^{u^{n}} u_{t}+u_{x x}+u_{x x x x}+(\lambda-1) u_{x}^{2}-\sigma$ $u_{x x}^{2}=0$ When $n=1$. For $n=1$, the equation is

$$
\Delta: e^{u} u_{t}+u_{x x}+u_{x x x x}+(\lambda-1) u_{x}^{2}-\sigma u_{x x}^{2}=0 .
$$

The general infinitesimal generator is

$$
V=\tau(x, t, u) \partial x+\mu(x, t, u) \partial t+v(x, t, u) \partial u
$$

In order to find the symmetry algebra, we have to apply invariance condition that is

$$
V^{(n)}(\Delta) \equiv 0 \bmod (\Delta)
$$

on equation (21) where $V^{(4)}$ is the fourth-order prolongation of $V$ such that

$$
\begin{aligned}
\operatorname{Pr}^{(4)} V= & V+v^{x} \frac{\partial}{\partial u_{x}}+v^{t} \frac{\partial}{\partial u_{t}}+v^{x x} \frac{\partial}{\partial u_{x x}}+v^{x t} \frac{\partial}{\partial u_{x t}} \\
& +v^{t t} \frac{\partial}{\partial u_{t t}}+v^{x x x} \frac{\partial}{\partial u_{x x x}}+v^{x x t} \frac{\partial}{\partial u_{x x t}}+v^{x t t} \frac{\partial}{\partial u_{x t t}} \\
& +v^{t t t} \frac{\partial}{\partial u_{t t t}}+v^{x x x x} \frac{\partial}{\partial u_{x x x x}}+v^{x x x t} \frac{\partial}{\partial u_{x x x t}} \\
& +v^{x x t t} \frac{\partial}{\partial u_{x x t t}}+v^{x t t t} \frac{\partial}{\partial u_{x t t t}}+v^{t t t t} \frac{\partial}{\partial u_{t t t t}}
\end{aligned}
$$
(21)

After applying invariance condition (23) on equation

$u e^{u} v^{t}+v^{x x}+v^{x x x x}+2(\lambda-1) u_{x} v^{x}-2 \sigma u_{x x} v^{x x} \equiv 0 \bmod (\Delta)$,

where $v^{t}, v^{x}, v^{x x}$, and $v^{x x x x}$ from equation (7) such that

$$
\begin{gathered}
v^{t}=D_{t}\left(v-\tau u_{x}-\mu u_{t}\right)+\tau u_{x t}+\mu u_{t t}, \\
v^{x}=D_{x}\left(v-\tau u_{x}-\mu u_{t}\right)+\tau u_{x x}+\mu u_{x t}, \\
v^{x x}=D_{x}^{2}\left(v-\tau u_{x}-\mu u_{t}\right)+\tau u_{x x x}+\mu u_{x x t}, \\
v^{x x x x}=D_{x}^{4}\left(v-\tau u_{x}-\mu u_{t}\right)+\tau u_{x x x x x}+\mu u_{x x x x t},
\end{gathered}
$$

where $D_{x}$ and $D_{t}$ are total derivative.

Putting all the above in equation (25), we eliminate $u_{t}$ by using the relation $u_{t}=1 / e^{u}\left(\sigma u_{x x}^{2}-(\lambda-1) u_{x}^{2}-u_{x x}-u_{x x x x}\right)$ and get a polynomial equation containing the different differentials of $u$. Equating the coefficient of $u$ to zero, which are some derivatives of $\tau, \mu$, and $\nu$, it gives the total set of determining equations.

$\tau_{x}=\tau_{t}=\tau_{u}=0, \mu_{x}=\mu_{t}=\mu_{u}=0, v=0$,

that gives

$$
\tau(x, t, u)=c_{3}, \mu(x, t, u)=c_{1} t+c_{2}, v(x, t, u)=c_{1} .
$$

This implies that the Lie group (algebra) of infinitesimal generators of equation (21) is comprised of three vector fields:

$$
\begin{aligned}
& V_{1}=\partial_{u}+t \partial_{t}, \\
& V_{2}=\partial_{t}, \\
& V_{3}=\partial_{x} .
\end{aligned}
$$

The commutator table of the Lie group for equation (21) is given in Table 5 ,

The adjoint table of infinitesimal symmetries for equation (21) is given in Table 6,

In this case, we have three different Lie algebras.

Theorem 3. The algebra of symmetries of KuramotoSivashinsky type equation is

$$
e^{u^{n}} u_{t}+u_{x x}+u_{x x x x}+(\lambda-1) u_{x}^{2}-\sigma u_{x x}^{2}=0,
$$


TABLE 5: Commutator table.

\begin{tabular}{lccc}
\hline$[. ., .]$. & $V_{1}$ & $V_{2}$ & $V_{3}$ \\
\hline$V_{1}$ & 0 & $-V_{2}$ & 0 \\
$V_{2}$ & $V_{2}$ & 0 & 0 \\
$V_{3}$ & 0 & 0 & 0 \\
\hline
\end{tabular}

TABLE 6: Adjoint table.

\begin{tabular}{cccc}
\hline$[a d]$ & $V_{1}$ & $V_{2}$ & $V_{3}$ \\
\hline$V_{1}$ & $V_{1}$ & $V_{2} e^{\varepsilon}$ & $V_{3}$ \\
$V_{2}$ & $V_{1}-\varepsilon V_{2}$ & $V_{2}$ & $V_{3}$ \\
$V_{3}$ & $V_{1}$ & $V_{2}$ & $V_{3}$ \\
\hline
\end{tabular}

where it is two-dimensional abelian for all $n>1, n \varepsilon N$ and three-dimensional nonabelian for $n=1$.

Proof. The proof follows easily using Propositions 1 and $2 . \square$

Theorem 4. If $G_{s}^{i}(x, t, u)$ be the one parameter group generated by equation (28) then

$$
\begin{aligned}
& G_{s}^{1}(x, t, u)=\left(x, e^{s} t, u\right), \\
& G_{s}^{2}(x, t, u)=(x, t+s, u), \\
& G_{s}^{3}(x, t, u)=(x+s, t, u) .
\end{aligned}
$$

There will be a family of solutions to each one parameter subgroups of the full symmetry group of a system called group invariant solutions.

Theorem 5. If $u=f(x, t)$ is a solution of equation (21), so are the functions

$$
\begin{aligned}
& \tilde{u}^{1}=f\left(x, e^{-s} t\right), \\
& \tilde{u}^{2}=f(x, t-s), \\
& \tilde{u}^{3}=f(x-s, t),
\end{aligned}
$$

where $\tilde{u}^{i}=G_{s}^{i} * f(x, t), i=1,2,3$ and $s<<1$ is any positive number.

Proof. The one parameter Lie group of equation (21) is

$$
G_{s}^{1}:(x, t, u) \longrightarrow\left(x, e^{s} t, u\right),
$$

with the infinitesimal generator

$$
V_{1}=t \partial t+\partial u
$$

if $\tilde{u}^{1}(x, t)$ is any function then it transformed by $G_{s}^{1}$ as

$$
\begin{aligned}
& \tilde{u}^{1}=u, \\
& \tilde{u}^{1}=f(x, t),
\end{aligned}
$$

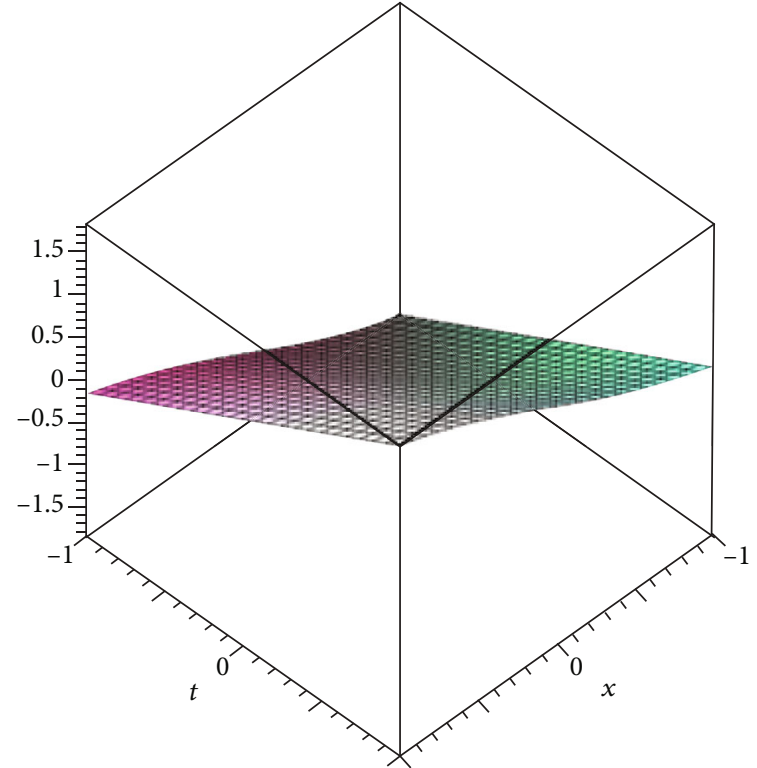

Figure 3: For $u^{1}(x, t)=\sin (x)+e^{-s}(t), s=0.00001$.

now

$$
(\tilde{x}, \tilde{t})=\left(x, e^{-s} t\right)
$$

therefore,

$$
\tilde{u}^{1}=f\left(x, e^{-s} t\right) .
$$

The graph for $\tilde{u}^{1}=f\left(x, e^{-s} t\right)$ is given in Figure 3. The one parameter Lie group of equation (21) is

$$
G_{s}^{2}:(x, t, u) \longrightarrow(x, t-s, u),
$$

with the infinitesimal generator

$$
V_{2}=t \partial t
$$

if $\tilde{u}^{2}(x, t)$ is any function then it transformed by $G_{s}^{2}$ as

$$
\begin{aligned}
& \tilde{u}^{2}=u, \\
& \tilde{u}^{2}=f(x, t),
\end{aligned}
$$

now

$$
(\tilde{x}, \tilde{t})=(x, t-s)
$$

therefore

$$
\tilde{u}^{2}=f(x, t-s)
$$

The graph for $\tilde{u}^{2}=f(x, t-s)$ is given in Figure 4 . The one parameter Lie group of equation (21) is

$$
G_{s}^{3}:(x, t, u) \longrightarrow(x-s, t, u),
$$




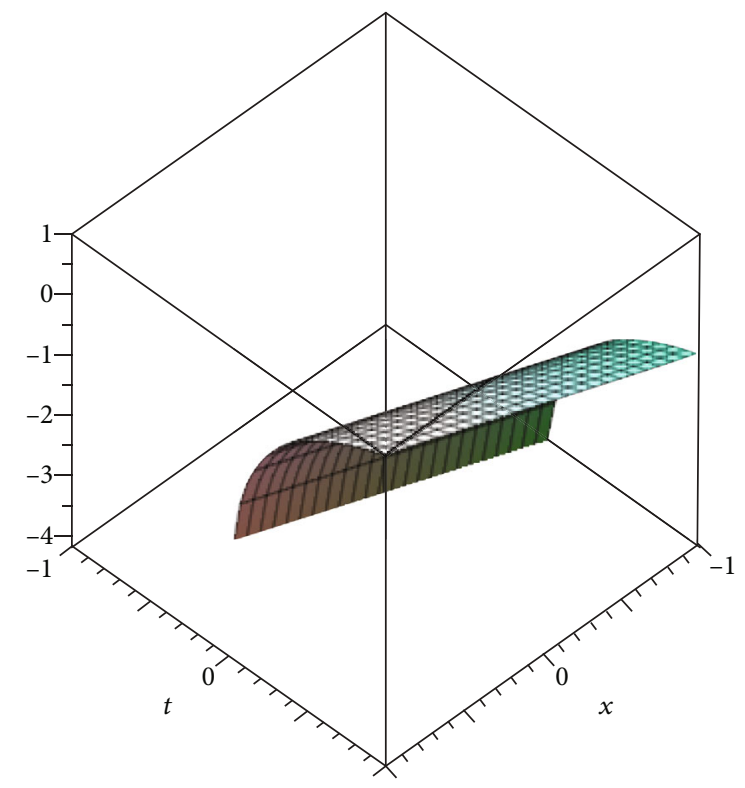

Figure 4: For $u^{1}(x, t)=\log (x+t)-s, s=0.00001$.

with the infinitesimal generator

$$
V_{3}=t \partial x
$$

if $\tilde{u}^{3}(x, t)$ is any function then it transformed by $G_{s}^{3}$ as

$$
\begin{aligned}
& \tilde{u}^{3}=u, \\
& \tilde{u}^{3}=f(x, t),
\end{aligned}
$$

now

$$
(\tilde{x}, \tilde{t})=(x-s, t)
$$

therefore

$$
\tilde{u}^{3}=f(x-s, t)
$$

The graph for $\tilde{u}^{3}=f(x-s, t)$ as a solution is given in Figure 5. $\square$

\section{Optimal System of Subalgebras}

This is remarkable that the Lie symmetry technique assumes a significant part to determine the solutions of PDEs as well as performing the symmetric reductions. Every combination (should be linear) of infinitesimal symmetries(generators) is a result of another infinitesimal symmetry(generator). As any transformation in the full symmetry groups plot a solution to another, it is sufficient to determine the invariant solution which are not related by transformations in the full symmetry group; this prompted the Optimal system [18, 19].

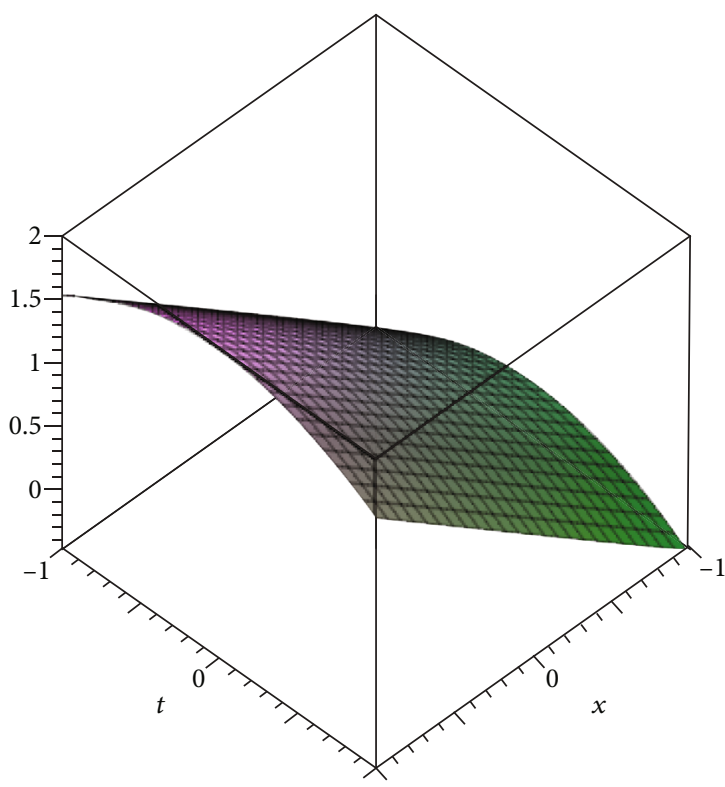

Figure 5: For $u^{3}(x, t)=(x-s)+\cos (t), s=0.00001$.

Theorem 6. A $1 D$ optimal system of equation (21) is given by those generated by

$$
\begin{aligned}
& Y_{1}=V_{1} \\
& Y_{2}=V_{2}, \\
& Y_{3}=V_{3}, \\
& Y_{4}=V_{1}+V_{3}, \\
& Y_{5}=V_{3}-V_{1} .
\end{aligned}
$$

Proof. Since the combination of vector field (infinitesimal generator) is also a vector field. Consider a linear combination $V$ of $V_{1}, V_{2}$, and $V_{3}$,

$$
V=\sum_{i=1}^{3}=b_{i} V_{i}
$$

a nonzero vector field. Here, for proof, we will improve as many of the coefficient $b_{i}{ }^{\prime} s$ as possible by using adjoint application on $\mathrm{V}$.

Case 1 . Firstly assume that $b_{3} \neq 0$ then

$$
V=b_{1} V_{1}+b_{2} V_{2}+V_{3}
$$

acting on $V$ with $\operatorname{Adj}\left(\exp \left(b_{2} / b_{1}\right) V_{2}\right)$ by using the adjoint table (adjoint Table 3)

$$
V^{\prime}=\operatorname{Adj}\left(\exp \frac{b_{2}}{b_{1}} V_{2}\right) V=b_{1} V_{1}+V_{3} .
$$

When $b_{1}>0$, then we get $Y_{4}$. When $b_{1}<0$, then we get $Y_{5}$. When $b_{1}=0$, then we get $Y_{3}$. 
Case 2. Let $b_{3}=0$ and $b_{1}=0$,

$$
V=b_{2} V_{2}
$$

when $b_{1}=1$ then we get $Y_{2}$, Let $b_{3}=0, b_{2}=0$ and $b_{1}$, then we get $Y_{1}$ There is no any more cases for consultation and the proof is complete.

\section{Lie Invariants and Similarity Solutions}

We can discover that the invariants correlate with the infinitesimal symmetries (28); they can be determined by solving the equations (by using characteristic method). For $V_{2}=\partial t$, the characteristic equation is $d x / 0=d t / 1=d u / 0$ and the corresponding invariants of this system $x=r$ and $u=w$.

We obtain a similar solution of the form $w=w(r)$, and we put it into equation (21) to obtain the form of the function $w$, and then, we conclude that $w=w(r)=w(x)$ solution of the following differential equation as similarity reduce equation:

$$
w_{r r}+w_{r r r r}+(\lambda-1) w_{r}^{2}-\sigma w_{r r}^{2}=0 .
$$

For other example, take $V_{3}=\partial x$; the characteristic equation for this has the form $d x / 1=d t / 0=d u / 0$ so the corresponding invariants are $t=r$ and $u=w$.

Taking into account the last invariants, the following similarity solution is obtained $w=w(r)=w(t)$ where the solution satisfied the similarity reduce equation:

$$
e^{w} w_{r}=0
$$

\section{Conclusions}

The present paper addresses Lie symmetries for some general cases of modified one-dimensional KuramotoSivashinky equation (MKS) as well as its similarity solutions using a symmetry operator. In Section 2, we discussed general results for Lie algebras for some general cases of MKS and provide a comparison between them and obtained some general results. In Section 3, we find the optimal system for (MKS). In the last section, we obtained similarity solutions and Lie invariants.

Remarks. It is worthmentioning that $f(u)$ can be any arbitrary function. For other similar functions chosen as $f(u)$, the procedure for symmetry analysis can be very tedious and symmetry algebra can be different.

\section{Data Availability}

Data sharing is not applicable to this article as no dataset was generated or analyzed during the current study

\section{Conflicts of Interest}

The authors declare that they have no conflicts of interest.

\section{Acknowledgments}

This research is partially supported by the Higher Education Commission, Pakistan.

\section{References}

[1] A. J. Bernoff and A. L. Bertozzi, "Singularities in a modified Kuramoto-Sivashinsky equation describing interface motion for phase transition," Physica D: Nonlinear Phenomena, vol. 85, no. 3, pp. 375-404, 1995.

[2] G. W. Bluman and J. D. Cole, "The general similiarity solution of the heat equation," Applied Mathematics and Mechanics, vol. 18, no. A969X, pp. 1025-1042, 1969.

[3] G. Cai, Y. Wang, and F. Zhang, "Nonclassical symmetries and group invariant solutions of Burgers-Fisher equation," World Journal of Modelling and Simulation, vol. 3, no. 4, pp. 305309, 2007.

[4] E. Buckwar and Y. Luchko, "Invariance of a partial differential equation of fractional order under the Lie group of scaling transformations," Journal of Mathematical Analysis and Applications., vol. 227, no. 1, pp. 81-97, 1998.

[5] M. Nadjafikhah and S. Dodangeh, "Lie symmetries and exact solutions for the one-dimensional Kuramoto-Sivashinsky equation," Applied Sciences, vol. 1, p. 22, 2020.

[6] F. Yan, C. Hua, H. Liu, and Z. Liu, "Lie symmetry analysis and exact solutions for the coupled Kuramoto-Sivashinsky equations," in 2011 Fourth International Workshop on ChaosFractals Theories and Applications, Hangzhou, China, October 2011.

[7] M. Nadjafikhah and F. Ahangari, "Symmetry reduction of two-dimensional damped Kuramoto-Sivashinsky equation," Communications in Theoretical Physics, vol. 56, no. 2, pp. 211-217, 2011.

[8] R. K. Gazizov and N. H. Ibragimov, "Lie symmetry analysis of differential equations in finance," Nonlinear Dynamics, vol. 17, no. 4, pp. 387-407, 1998.

[9] W. Shu-Yong and M. Feng-Xiang, "Form invariance and Lie symmetry of equations of non-holonomic systems," Chinese Physics, vol. 11, no. 1, pp. 5-8, 2002.

[10] Y. Bozhkov and S. Dimas, "Group classification and conservation laws for a two-dimensional generalized KuramotoSivashinsky equation," Nonlinear Analysis: Theory, Methods and Applications, vol. 84, pp. 117-135, 2013.

[11] M. Nadjafikhah and F. Ahangari, "Lie symmetry analysis of the two-dimensional generalized Kuramoto-Sivashinsky equation,” Mathematical Sciences, vol. 6, no. 1, pp. 1-7, 2012.

[12] K.-S. Chou, "On a modified Kuramoto-Sivashinsky equation," Differential and Integral Equations, vol. 15, no. 7, pp. 863-874, 2002.

[13] Z. Csahók, C. Misbah, and A. Valance, “A class of nonlinear front evolution equations derived from geometry and conservation," Physica D: Nonlinear Phenomena, vol. 128, no. 1, pp. 87-100, 1999.

[14] S. Childress and E. A. Spiegel, "Pattern formation in a suspension of swimming microorganisms: nonlinear aspects," in $A$ Celebration of Mathematical Modeling, pp. 33-52, Springer, Dordrecht, 2004.

[15] H. Lai and C. Ma, "Lattice Boltzmann method for the generalized Kuramoto-Sivashinsky equation,” Physica A, vol. 388, no. 8, pp. 1405-1412, 2009. 
[16] R. Conte, Exact Solutions of Nonlinear Partial Differential Equations by Singularity Analysis, Lecture Notes in Physics, Springer, Berlin, 2003.

[17] S. Saprykin, E. A. Demekhin, and S. Kalliadasis, "Two-dimensional wave dynamics in thin films. I. Stationary solitary pulses," Physics of Fluids, vol. 17, no. 11, pp. 117105-117116, 2005.

[18] M. Munir, M. Athar, S. Sarwar, and W. Shatanawi, "Lie symmetries of generalized equal width wave equations," AIMS Mathematics, vol. 6, no. 11, pp. 12148-12165, 2021.

[19] P. J. Olver, Applications of Lie Groups to Differential Equations, Springer, New York, 1986. 